\title{
Genome-wide identification, classification and expression profile analysis of the HSF gene family in Hypericum perforatum
}

\author{
Li Zhou ${ }^{1}$, Xiaoding Yu ${ }^{1}$, Donghao Wang ${ }^{1}$, Lin Li $^{1}{ }^{1}$, Wen Zhou ${ }^{1}$, Qian Zhang ${ }^{1}$, Xinrui Wang ${ }^{1}$, Sumin Ye ${ }^{1}$, Zhezhi \\ Wang ${ }^{\text {Corresp. } 1}$ \\ 1 National Engineering Laboratory for Resource Development of Endangered Crude Drugs in Northwest China, Key Laboratory of the Ministry of Education
for Medicinal Resources and Natural Pharmaceutical Chemistry, College of Life Sciences, Shaanxi Normal University, Xi'an, Shaanxi, China \\ Corresponding Author: Zhezhi Wang \\ Email address: zzwang@snnu.edu.cn
}

Heat shock transcription factors (HSFs) are critical regulators of plant responses to various abiotic and biotic stresses, including high temperature stress. HSFs are involved in regulating the expression of heat shock proteins (HSPs) by binding with heat stress elements (HSEs) to defend against high-temperature stress. The $H$. perforatum genome was recently fully sequenced; this provides a valuable resource for genetic and functional analysis. In this study, 23 putative HpHSF genes were identified and divided into three groups $(A, B$, and $C)$ based on phylogeny and structural features. Gene structure and conserved motif analyses were performed on HpHSFs members; the DNA-binding domain (DBD), hydrophobic heptad repeat (HR-A/B), and exon-intron boundaries exhibited specific phylogenetic relationships. In addition, the presence of various cis-acting elements in the promoter regions of $\mathrm{HpHSFs}$ underscored their regulatory function in abiotic stress responses. RT-qPCR analyses showed that most HpHSF genes were expressed in response to heat conditions, suggesting that HpHSFs play potential roles in the heat stress resistance pathway. Our findings are advantageous for the analysis and research of the function of HpHSFs in high temperature stress tolerance in $H$. perforatum. 
1 Genome-wide identification, classification, and

2 expression profile analysis of the HSF gene family in

3 Hypericum perforatum

4

5 Li Zhou, Xiaoding Yu, Donghao Wang, Lin Li, Wen Zhou, Qian Zhang, Xinrui Wang, Sumin

6 Ye, Zhezhi Wang*

7

8

9

10

11

12

13

14

15

16

17

18

19

20

21

22

23

24

25

26

27

28

29

30

*Corresponding Author:

Zhezhi Wang

Shaanxi Normal University, Xi'an, Shaanxi, 710100, China

Email address: zzwang@snnu.edu.cn

\section{Abstract}

Heat shock transcription factors (HSFs) are critical regulators of plant responses to various abiotic and biotic stresses, including high temperature stress. HSFs are involved in regulating the expression of heat shock proteins (HSPs) by binding with heat stress elements (HSEs) to defend against high-temperature stress. The H. perforatum genome was recently fully sequenced, this provides a valuable resource for genetic and functional analysis. In this study, 23 putative $H p H S F$ genes were identified and divided into three groups (A, B, and C) based on phylogeny and structural features. Gene structure and conserved motif analyses were performed on HpHSFs members; the DNA-binding domain (DBD), hydrophobic heptad repeat (HR-A/B), and exon-intron boundaries exhibited specific phylogenetic relationships. In addition, the presence of various $c i s$-acting elements in the promoter regions of $H p H S F$ s underscored their regulatory function in abiotic stress responses. RT-qPCR analyses showed that most $H p H S F$ genes were expressed in response to heat conditions, suggesting that HpHSFs play potential roles 
31 in the heat stress resistance pathway. Our findings are advantageous for the analysis and research

\section{Introduction}

High temperature as an abiotic stress triggered by global warming is largely the result of deforestation and increases in atmospheric $\mathrm{CO}_{2}$ concentrations. Global warming has caused worldwide declines in the yield of crops including wheat, rice, maize, and soybean, which are the most widely consumed staple foods in the world and feed over 50\% of humanity (Mittler et al. 2012; Sadok et al. 2020; Song et al. 2018; Zhu et al. 2019). High temperature has a pernicious impact on plants, such as oxidative stress and membrane permeabilization, due to effects on photosynthetic efficiency and decreased grain weight. Plants deploy several responses to mitigate high temperature stress. The physiological and biochemical processes of stomatal development, shade avoidance response, antioxidant defense, and selective autophagy play important roles in adaptation to high temperature stress. These processes are regulated by essential genes and specific transcriptional factors that are involved in modulating mechanisms and the alleviation of hightemperature stress (Samakovli et al. 2020; Thirumalaikumar et al. 2020). Under heat stress, heat shock transcription factors (HSFs) can activate the rapid accumulation and expression of heat shock proteins (HSPs) to reduce heat-related damage such as electrolyte leakage, overproduction of reactive oxygen species (ROS) and oxidative damage (Bokszczanin et al. 2013). Many HSPs play a critical role in protecting plants from heat-induced damage as well as in protein folding, aggregation, degradation, and intracellular distribution (Lin et al. 2011; Mittler et al. 2012). In the heat shock reaction process, HSFs regulate the expression of heat stress-inducible genes by recognizing the heat stress elements (HSEs) present in the promoter regions of HSP genes (Scharf et al. 2012). Specifically, HSFs utilize their oligomerization domains to form trimmers and exert their functions as sequence-specific trimeric DNA-binding proteins. Previous studies have shown that transcription activation in vivo requires HSEs in HSF protein binding. The HSF recognizes a typical 5 bp sequence, 5-nGAAn-3, which forms at least three contiguous inverted repeats in the downstream HSP promoter (Saha et al. 2019). The highly conserved DNA-binding domain (DBD) in the N-terminus includes one three-helical bundle $(\alpha 1, \alpha 2, \alpha 3)$ and one antiparallel four-stranded $\beta$-sheet $(\beta 1, \beta 2, \beta 3, \beta 4)$ to form a helix-turn-helix structure. The DBD domain is required for HSE specific binding to regulate the expression of downstream genes (Guo et al. 2016). The oligimerisation domain (OD), also known as the HR-A/B region, has the characteristics of a coiledcoil structure and plays a role in transcription factor activity. It is mainly located at the C-terminal end of the HSF and connected to the DBD through a flexible linker comprising a heptad pattern of hydrophobic amino acid residues (Lin et al. 2015). A nuclear localization signal (NLS) is also present at the C-terminal of the HR-A/B region, consisting of a cluster of basic amino acids rich in lysine and arginine residues, and is essential for nuclear import; some HSF genes also have a nuclear export signal (NES) in the C-terminus, which contains several leucine residues and is crucial for regulating the nucleocytoplasmic distribution of HSF proteins (Chidambaranathan et al. 2018). Some HSF proteins also have short peptide motifs (AHA motifs) close to the C-terminal 
70 for transcriptional activator functions (Kotak et al. 2004). Based on analysis of the conserved DBD

71 domain and HR-A/B regions, HSFs in plants are classified into three main classes (class A, B, and

72 C) (Nover et al. 2001). The number of amino acid residues connecting DBD to HR-A/B differs

73 among the three subgroups: Classes A, B, and C contain 9-39, 50-78, and 4-49 amino acid

74 residues, respectively (Miller \& Mittler 2006; Prandl et al. 1998). Moreover, the number of amino

75 acids linking HR-A and HR-B also varies considerably in different subgroups. There are 21 and 7

76 amino acid residues inserted into the HR-A/B region in class $A$ and class $\mathrm{C}$, respectively, whereas

77 this region in class B HSFs is compact, without insert sequences, between the heptad

78 repeats(Baniwal et al. 2004). Additionally, the AHA motifs, which function by binding

79 transcription protein complexes to activate the transcription of HSPs, are unique to class A

80 members (Scharf et al. 2012). Recently, HSF gene families were analyzed in different species,

81 including maize, rice, pepper, tomato, soybean, and flax. Genome-wide analysis indicated that

82 HSF proteins in various species may have different functions in reducing damage to high-

83 temperature stress and also provide resources for evolutionary analysis (Guo et al. 2015; Saha et

84 al. 2019; Yang et al. 2016).

Hypericum perforatum is an herbaceous perennial plant in the family Hypericaceae, the well-characterized secondary metabolites and pharmacological activities of which have attracted the attention of researchers (Galeotti 2017). Substances present in the extracts of $H$. perforatum include acyl-phloroglucinols, naphthodianthrones, xanthones, and flavonoids, and these pharmacological compounds have been demonstrated to have antiviral, antitumor,

90 antiinflammatory, antimicrobial, and antioxidant effects (Nahrstedt \& Butterweck 2010). However, $H$. perforatum production and quality are challenged by various environmental stresses, such as cold, high temperature, and drought (Lausen et al. 2020; Skyba et al. 2012; Zobayed et al. 2005). Therefore, it is important to characterize the stress-resistance genes of $H$. perforatum. In the current study, we identified $23 \mathrm{HpHSF}$ genes and analyzed their physical and chemical characteristics, conserved domains, gene structures, evolutionary relationships, and cisacting elements. Moreover, we explored expression profiles across four different tissues. In conclusion, this study provides a foundation for improved exploration of $H p H S F$ gene function in H. perforatum.

\section{Materials \& Methods}

\section{Plant materials and treatment}

102 Seeds of $H$. perforatum preserved in our laboratory were germinated and grown on a seedling 103 bed in a greenhouse $\left(25 \pm 2{ }^{\circ} \mathrm{C}\right.$, natural lighting $)$. Humidity was maintained at $60 \%-80 \%$. Twomonth-old $H$. perforatum seedlings were transferred to an incubator maintained at $42{ }^{\circ} \mathrm{C}$ for heat stress treatments, and five time points $(0,1,3,6$, and $12 \mathrm{~h})$ were selected for sample collection. 
107 treatment. In addition, different tissue samples, including flowers, leaves, stems, and roots, were

108

109

\section{Identification of HpHSF members}

111 The whole genome sequences of the HSF proteins in H. perforatum were detected and assembled taken from two-year-old plants. All samples were collected in three replicates, and the samples were immediately immersed in liquid nitrogen and stored at $-80^{\circ} \mathrm{C}$ for RNA isolation. by our laboratory (Zhou 2020). For HSF identification, the conserved amino acid sequence of DNA-binding domains (Pfam: PF00447) was used to search the H. perforatum genome.

Moreover, the HSF sequences of Capsicum annuum L. (pepper), Vitis vinifera L. (grape), and Arabidopsis thaliana obtained from plantTFDB (http://planttfdb.cbi.pku.edu.cn) were used as BLAST queries against the $H$. perforatum genome. All output genes with default were searched for conserved DNA-binding domains using Interpro (http://www.ebi.ac.uk/interpro/) and SMART (http://smart.embl-heidelberg.de/). In addition, the candidate genes were analyzed using MARCOIl (http://toolkit.tuebingen.mpg.de/marcoil) to retain genes with a coiled-coil structure. The detected genes are listed in Supplementary Table 1.

\section{Phylogenetic relationship analysis and sequence analysis}

Full-length amino acid sequences of HSF from A. thaliana, C. annuum L., V. vinifera L. (grape), and $H$. perforatum were aligned using Clustal $\mathrm{X}$; the extension penalty and opening penalty of gap were 0.2 and 10, respectively. The cut-off for delay divergent sequences was set to $40 \%$. Residue-specific and hydrophilic penalties were applied in alignment. Then, the phylogenetic tree was inferred using MEGA 6.0. The statistical method used was the neighbor-joining (NJ) method, and the test of phylogeny was based on the bootstrap method with 1000 bootstrap replicates and pairwise deletion. The amino acid substitution model used was the p-distance model. Parameters including molecular weight, isoelectric point, aliphatic index, instability index, percentage of negatively/positively charged residues, and GRAVY of HpHSF proteins were displayed using the ExPASy database (https://www.expasy.org/). Furthermore, conserved motifs in HpHSF proteins were searched using Heatster (Heatster, https://applbio.biologie.unifrankfurt.de/hsf/heatster/) and the exon/intron organization of HpHSF proteins was obtained using the Gene Structure Display Server program (GSDS, http://gsds.cbi.pku.edu.cn/). The cisacting elements of $1.5 \mathrm{~kb}$ upstream sequences of the transcription initiation site in the promoter region of $H p H S F$ genes were analyzed using PlantCARE

(http://bioinformatics.psb.ugent.be/webtools/plantcare/html/). SWISS-MODEL server (https://swissmodel.expasy.org) programs were used to build and generate the three-dimensional structures of the HSF proteins.

\section{Isolation of RNA and cDNA synthesis}

Total RNA from $H$. perforatum samples was isolated using the HiPure Total RNA Mini Kit following the manufacturer's protocol (Magen, China). The concentration of the isolated total RNA was determined using a NanoDrop 2000c spectrophotometer (Thermo Scientific, USA), 
144 and the integrity of the RNA was directly quantified by running agarose gel $(1 \% \mathrm{w} / \mathrm{v})$ at $150 \mathrm{~V}$

145 for $10 \mathrm{~min}$. One microgram of RNA was used for first strand cDNA synthesis using

146 PrimeScriptTM RT Reagent Kit (TaKaRa, China) according to the manufacturer's instructions.

147 Primer design and $\mathbf{q R T}-\mathbf{P C R}$ analysis

148 The primers for the $23 \mathrm{HpHSF}$ genes were designed by GenScript (https://www.genscript.com).

149 The parameters were: PCR amplicon size range: 100 - 180; primer Tm: minimum, optimum, and

150 maximum: $59.5^{\circ} \mathrm{C}, 60^{\circ} \mathrm{C}, 60.5^{\circ} \mathrm{C}$, respectively; probe $\mathrm{Tm}$ : minimum, optimum, and maximum:

$15162^{\circ} \mathrm{C}, 66^{\circ} \mathrm{C}$, and $70^{\circ} \mathrm{C}$, respectively. The specificity of the primers was determined using Bioedit

152 by searching the primers given by GenScript against the H. perforatum genome (Supplementary

153 Table 2). In addition, qRT-PCR was performed on the LightCycler 96 system (Roche

154 Diagnostics $\mathrm{GmbH}$ ) using the ChamQTM SYBR ${ }^{\circledR}$ qPCR Master Mix (Vazyme, Nanjing, China)

155 following the manufacturer's instructions. HpActin-2 was used as an internal control (Zhou

156 2019). The final cycle threshold $(\mathrm{Ct})$ values were the mean of three values for each sample and

157 three technical replicates, and the $2-\Delta \Delta \mathrm{Ct}$ method was used to analyze the relative changes in

158 gene expression (Livak \& Schmittgen 2001). Data were analyzed using one-way ANOVA in the

159 GraphPad Prism software $(*, \mathrm{P}<0.05$; **, $\mathrm{P}<0.01$; **, $\mathrm{P}<0.001)$. qRT-PCR was performed

160 with three biological replicates for each sample, and each sample consisted of three technical

161 replicates. The primers for the $H p H S F$ genes used for qRT-PCR analyses are listed in

162 Supplementary Table 1.

163

164 Results

165 Identification and isolation of $\boldsymbol{H S F}$ genes in $\boldsymbol{H}$. perforatum

166 Twenty-three genes were identified as members of the HSF transcription factor family in $H$.

167 perforatum based on a conserved DBD domain search and coiled-coil structure detection. These

168 genes were named 'HpHSF' with consecutive numbers. More detailed information about

169 HpHSF01 to HpHSF23 is shown in Table 1. The identified HpHSF encoded 188-501 amino

170 acids (average $345 \mathrm{aa}$ ), and molecular weights (MW) ranged from 21.72. to $54.91 \mathrm{kDa}$ (average

$17139.15 \mathrm{kDa}$ ). The isoelectric points (pI) of HpHSFs varied from 4.79 to 8.86. Of the $23 \mathrm{HpHSF}$

172 genes, the percentages of negatively charged residues (ASP + Glu) (n.c.r.) and positively charged

173 residues (Arg + Lys) were $11.0 \%-17.6 \%$ and $8.4 \%-15.8 \%$, respectively. According to the

174 instability index analysis, all the HpHSF proteins were found unstable. In addition, the aliphatic

175 index (A.I.) ranged from 54.52 to 76.18 , and the grand average of hydropathicity (GRAVY)

176 ranged from -0.826 to -0.523 .

\section{Conserved domains of $\boldsymbol{H p H S F}$}


178 The DBD and HR-A/B conserved domains were observed in the all of the HpHSF genes to

179

180

181

182

183

184

185

186

187

188

189

190

191

192

193

194

195

196

197

198

199

200

201

202

203

204

205

206

207

208

209

210

211

212

213

214

215

reveal the sequence of conserved regions between members of the HpHSFs; multiple alignments of $23 H p H S F \mathrm{~s}$ were obtained using DNAMAN. The DBD domain close to the N-terminal was highly conserved (Fig. 1). The secondary structure prediction showed that the majority of the DBD domains consist of a four-stranded antiparallel $\beta$-sheet and three $\alpha$-helices $(\alpha 1-\alpha 3)$. In addition, MARCOIL was used for predicting the coiled-coil structure, which is characteristic of the HR-A/B regions adjacent to the DBD domain in the C-terminal. The 23 candidate HpHSF protein sequences were all determined to have a coiled-coil structure. The multiple alignment results of the HR-A/B regions showed that the HpHSF protein family could be divided into three classes based on the insertion amino acid residues between the A and B parts of the HR-A/B motif (Fig. 2).

\section{Phylogenetic relationships among $\mathrm{HpHSF}$ genes}

To investigate the evolutionary relationships of the $H p H S F$ genes, a total of $88 H S F s$, comprising 21 from Arabidopsis, 25 from pepper, 19 from grape, and 23 from $H$. perforatum were used for phylogenetic tree construction using MEGA6.0. HSFs were clearly classified into three main groups, namely HSF A, B, and C (Fig. 3). HpHSF A was the largest group, representing $52.2 \%$ of the total HpHSFs; the second was HpHSF B, which represented 39.1\%; and HpHSF C was the smallest group, which represented $8.7 \%$. In addition, HpHSF A is classified into 9 subgroups (A1-A9) and includes 12 members (HpHSF07, HpHSF18, HpHSF12, HpHSF11, HpHSF16, HpHSF 21, HpHSF 17, HpHSF02, HpHSF23, HpHSF13, HpHSF10, HpHSF20); HpHSF B is further divided into 5 subgroups (B1-B5) consisting of nine members (HpHSF01, HpHSF03, HpHSF04, HpHSF 05, HpHSF06, HpHSF14, HpHSF15, HpHSF19, HpHSF22), while HpHSFC only contained HpHSF 08 and HpHSF09 in one subgroup. All of the HpHSFs in the phylogenetic tree were consistent with the classifications obtained from the HEATSTER database. HpHSF proteins were not clustered in A2, A7 and A9 sub-groups.

\section{Gene Structures analysis, Conserved Motifs, and Protein Modeling of HpHSFs}

The gene structures of $H p H S F s$ were investigated through an analysis of the intron/exon boundaries, as shown in Figure 4A. HpHSF 20 comprised three exons, and HpHSF03 comprised four exons, except for the aforementioned two HpHSFs, all the other $21 \mathrm{HpHSFs}$ contained two exons and one intron. The intron phases of HpHSFs were 0, except for phase 1 in HpHSF20 and phase 2 in $H p H S F 03$. In conclusion, the gene structure was conserved among the $23 \mathrm{HpHSF}$ members.

In addition, the conserved motifs and phylogenetic relationships of all $23 \mathrm{HpHSF}$ proteins were revealed via a systematic examination (Table 2 and Fig. 4B). The HSF domains DBD, OD (HRA/B), NLS, RD (Repressor Domain), AHA, and NES were found in HpHSF protein sequences. Twelve, nine, and two HpHSF proteins were classified in subclasses A, B, and C, respectively. The HpHSF proteins in subclass A were characterized by DBD at the N-terminus followed by the HR-A/B motif. NLS, AHA, and NES were found in partial subclass A HSF proteins. The RD

Peer] reviewing PDF | (2020:09:52717:1:1:NEW 24 Jan 2021) 
216 motif was only found in subclass B HpHSF sequences. Subclass C contained DBD, HR-A/B, and 217 NLS, which were considered to be highly conserved motifs in HpHSF proteins. The HpHSF 218 proteins were modeled using the SWISS-MODEL program (Fig. 5). A Drosophila heat shock 219 transcription factor was used as a template, and the template model was taken from the Protein 220 Data Bank (SMTL ID: 1hkt.1). The HpHSFs shared approximately 40\% sequence similarity and

221 222

223

224

225

226

227

228

229

230

231

232

233

234

235

236

237

238

239

240

241

242

243

244

245

246

247

248

249

250

251

252

253

$30 \%$ query coverage. The start position of the $\alpha 1$ DBD domain was notated.

\section{Cis-acting elements analysis in the promoter regions of HpHSF genes}

We searched for potential cis-acting elements in the $1.5 \mathrm{~kb}$ upstream sequences of the translation initiation codons of HpHSFs in the PlantCARE database, and the results revealed the presence of various cis-elements in the $5^{\prime}$ flanking regions associated with stress, hormones, and development (Ning et al., 2017). In stress-related cis-acting elements, some elements related to various stresses, such as light, low/high temperature, drought, anaerobic induction, and wounds were found in a large number of $H p H S F$ genes, including heat-shock response elements (HSEs), TC-rich repeats, Myb-binding DNA sequences (MBSs), anaerobic induction elements (AREs), and low temperature range (LTR) (Fig. 6, Supplementary Table 4). In addition, several hormonerelated cis-acting elements were observed in the promoters: ABA-responsive elements (ABREs), MeJA responsive elements (TGACG-motif/CGTCA-motif), ethylene-responsive element (ERE), auxin-responsive elements (TGA-elements), and salicylic acid responsive elements (TCAelements) were detected in the promoters of 19, 17, 13, 13 and, and $7 \mathrm{HpHSF}$ s, respectively. These findings suggested that the $H p H S F$ genes might be involved in multiple transcriptional regulation mechanisms for plant growth and stress responses.

\section{Expression profiles of $\mathrm{HpHSFs}$ across different tissues}

Based on the $H$. perforatum genes against RNA-seq data from four tissues - roots, stems, leaves, and flowers, a heat map of the transcription patterns of the HpHSF family was generated to explore the transcription patterns of $H p H S F$ genes. RNA-seq data could be searched from the Sequence Read Archive (SRA-NCBI) with accession numbers SRR8438983 (flower), SRR8438984 (leaf), SRR8438985 (stem), and SRR8438986 (root). According to the FPKM values, the expression profiles of the HpHSF gene differed considerably in the four samples (Fig. 7). Among class A members, HpHSF 12, HpHSF 18, and HpHSF 13 were expressed at high levels, while $H p H S F 02$ and $H p H S F 23$ were expressed at relatively low levels or were not detected. Moreover, the expression of HpHSF 11, HpHSF 18, HpHSF 13, and HpHSF07 in leaves was higher than that in other tissues. Among the class B families, HpHSF 15 was expressed at significantly high abundances in all tissues compared with other genes. HpHSFO3 and HpHSF22 were expressed at very low levels or not expressed at all. In general, members of the class B family were expressed at higher levels in the root than in other tissues, except $H p H S F 01$, as well as the two members of class $\mathrm{C}$, implying their critical roles in roots.

\section{Expression analysis of $\mathrm{HpHSF}$ genes under heat stress treatment}

Peer] reviewing PDF | (2020:09:52717:1:1:NEW 24 Jan 2021) 
254 HSF genes were found to play an important role in plant thermotolerance. In our study, the 255 expression patterns of the HpHSF gene family were determined using qRT-PCR to demonstrated 256 how HSF genes respond to heat stress. As shown in Figure 8, the expression of HpHSF2, 12, and 25721 did not significantly change with heat stress. HpHSF03, 11, 18 and 22 were repressed after 258 heat stress treatment, and the remaining HpHSFs were up-regulated to varying degrees.

259 Noticeably, the expression of HpHSF 10 increased dramatically, and was approximately 300 260 times higher at $3 \mathrm{~h}$ than the levels in the control, indicating that HpHSF 10 is involved in the 261 pathway of heat stress response. In addition, the expression of $H p H S F 1,14,20$, and 23 also 262 changed considerably, and these genes are thus worthy of further consideration.

\section{Discussion}

264 Temperature is a key environmental factor affecting several physiological pathways in plants.

265 Secondary metabolite production determines the immunologic defense and economic value of $H$. 266 perforatum, which is a medicinal plant. The concentration of hypericin and pseudohypericin in $267 H$. perforatum is closely related to temperature. The heat tolerance and photosynthetic rates of $H$. 268 perforatum are both significantly reduced at high temperatures, and the total hypericin content 269 (hypericin + pseudohypericin) is lower following high temperature treatment (Zobayed et al. 270 2005). Heat stress has been demonstrated to be detrimental in other species, seed yield is reduced 271 following exposure for five days to high temperatures in flax (Gusta et al. 1997). It is thus of 272 great importance to study how medicinal plants respond to high temperature stress with regard to 273 growth, metabolism, photosynthesis, and even global climate. The HSF gene family plays an 274 important role in plant adaptations to various biotic or abiotic stresses, especially high 275 temperature stress. HSFs regulate HSPs as a partner at the genetic and transcriptional level to 276 improve high-temperature stress tolerance (Wang et al. 2004). In this study, the identification 277 and characteristics of $23 \mathrm{HSF}$ genes were investigated based on the $H$. perforatum genome 278 database, and the expression profiles of these 23 genes were analyzed to explore their functions 279 in the heat stress response in H. perforatum. The number of HpHSFs was low in comparison 280 with numbers identified for other species; the 23 non-redundant complete genes in $H$. perforatum 281 were fewer than those in G. raimondii (57), Salix purpurea (48), and Linum usitatissimum (34). 282 Overall, the isolation and identification of these $H S F$ genes are helpful for illustrating the 283 molecular genetic basis of $H$. perforatum. The expression patterns of $H p H S F \mathrm{~s}$ in four tissues and 284 response to heat stress at $42{ }^{\circ} \mathrm{C}$ suggested that the $H S F$ gene family was ubiquitously expressed, 285 and several $H p H S F$ genes could play important roles in adaptation to environmental stress.

286 The essential structures and motifs of $23 \mathrm{HpHSF}$ genes and amino acids were identified. The 287 conversed motifs of HpHSF protein, DBD, OD (HR-A/B), NLS, NES, and RD, were annotated. 288 The DBD domain consists of approximately 100 amino acid residues that are highly conserved in 289 yeast, plants, and mammals(Schultheiss et al. 1996). Similar to the results of previous studies, 290 our findings showed that many sequences are conserved based on phylogenetic relationships in 291 Arabidopsis, pepper, grape, and $H$. perforatum and the coiled-coil structure of HR-A/B region 292 prediction. The HpHSF genes were classified into three classes (A, B, and C). Classes A and B 
293 were further divided into 9 (A1-A9) and 5 (B1-B5) subclasses, respectively. The number of class 294 A HSF genes varies in plants - 15 in Arabidopsis and maize, 13 in rice and mung, and 16 in 295 soybean. There were nine class B HSFs in H. perforatum. The number of class B HSFs identified 296 in plants is 10 in mungbean, 8 in rice, 7 in maize, and 5 in Arabidopsis. Most of the subclasses 297 are shared among several species but are not identical. The subclasses A2, A7, and A9 identified 298 in our study have been reported in some species, such as Arabidopsis and Arachis(Wang et al. 299 2017), but not in H. perforatum. It was hypothesized that elimination of introns, exon shuffling, 300 and generations of exons might cause altered grouping in the phylogeny(Nover et al. 2001). 301 Overall, these observations suggested the functional conservation and divergence of $H S F$ genes 302 among different plants.

303 HSF protein is involved in abiotic stress responses and hormone signaling in plants(Huang et al. 304 2015; Zhang et al. 2015). The cis-acting elements in the promoter region can regulate the 305 transcription activity of the corresponding genes. Research on the detection of cis-acting 306 elements could facilitate our understanding of the function and expression profiles of genes 307 (Fragkostefanakis et al. 2015; Wang et al. 2017). The promoter region of the $\mathrm{HpHSF}$ gene family 308 members contains various elements related to growth and development, hormone responses, and 309 stress responses. The numbers and types of elements vary among the HpHSF promoters, and 310 overlapping phenomena existed in different genes, implying that the members of the family may 311 regulate a variety of abiotic stresses and plant hormone signaling pathways simultaneously. This 312 reflects the diversity and complexity of the biological functions of the $H p H S F$ gene family.

313 Gene expression profiles in different tissues are usually closely correlated with their functions in 314 organ development(Guo et al. 2008). In this study, the expression patterns of $H p H S F$ genes in 315 four different tissues were investigated. Remarkably, the expression of HSF 15 was found to be 316 highest among all genes in all four tissues. Each gene was expressed differently in the four 317 tissues, such as $H S F 10$, which had the highest expression in roots but the lowest expression in 318 flowers, and HSF18, the expression of which was higher in leaves than in other tissues, 319 indicating their potential function in roots and leaves, respectively. All these $H p H S F$ genes play 320 roles in different tissues to ensure the normal development of plants. The low expression in 321 322 323 certain organs of some HSFs does not mean that they have no function in these organs. Tissuespecific expression patterns of identified $\mathrm{HpHSF}$ genes indicate that HpHSFs are widely involved in the growth and development of various tissues, indicating an important role for studying the functions of $\mathrm{HpHSF}$ genes in $\mathrm{H}$. perforatum developmental biology.

Plant HSFs play a central role in eliciting the expression of genes encoding heat shock proteins (HSPs) or other stress-inducible genes(Nishizawa-Yokoi et al. 2009; Scharf et al. 2012), which are important for plant tolerance to heat or other stress conditions. According to previous reports, the genome-wide expression profile suggested that several $H S F$ genes are transcribed at relatively high levels during heat stress (Chung et al. 2013; Giorno et al. 2012). 
330 In this study, the $23 H p H S F$ genes with specific sequence features and amino acid motifs were

331

332

333

334

335

336

337

338

339

340

341

342

343

\section{Conclusions}

345

346

347

348

349

350

351

352

353

354

355

356

357

358

359

360

361

362

363

364

365

366

367 temperature tolerance. stress responses. encouragement.

\section{References}

identified. Base on the motifs, the $H p H S F$ genes were phylogenetically divided into three broad groups. The abiotic stress-related cis-acting elements were identified in the promoter of $\mathrm{HpHSF}$ genes. The expression of $23 \mathrm{HpHSF}$ genes in different tissues and distinct patterns during heat treatment was performed. Among these genes, $14 \mathrm{HpHSF}$ genes were upregulated ( $>2$-fold) and $4(H p H S F 3,11,18,22)$ were downregulated during the heat stress treatment. Specifically, HpHSF 10 was the most strongly induced ( 2300 -fold) in response to heat stress; HSF 20 expression was more than 90 folds that of the control after heat treatment; the levels of HSF14, $H S F 15$, and $H S F 23$ were about 20 times higher than those in the control group, indicating that these $H p H S F$ genes were very sensitive with a strong heat stress response. These genes play an important role in regulating the response of $H$. perforatum to heat stress and warrant further attention and exploration. All the systematic and phylogenetically analysis of $H p H S F$ genes contributed to the genomic improvement and medical values of $H$. perforatum for high

In conclusion, a comprehensive analysis of the $H p H S F$ gene family with regard to the genomic structures, conserved motifs, phyletic evolution, cis-acting elements, and expression patterns was performed in this work. Overall, the bioinformatic analyses and expression profile studies of HSFs are helpful in understanding the important role of HSFs in H. perforatum's response to heat stress and providing the foundation for exploring methods to understand and regulate these

\section{Acknowledgements}

I would like to express my gratitude to all the people who helped me with writing this article, especially my supervisor, Prof. Wang, the completion of this thesis is inseparable from his valuable suggestions and patient guidance. I am also grateful to Lin $\mathrm{Li}$, Donghao Wang, Qian Zhang and Xiaoding $\mathrm{Yu}$ for their help in the design of the study, acquisition of data and writing of manuscripts. Finally, I am indebted to my beloved family for their consistent support and

Baniwal SK, Bharti K, Chan KY, Fauth M, Ganguli A, Kotak S, Mishra SK, Nover L, Port M, Scharf KD, Tripp J, Weber C, Zielinski D, and von Koskull-Doring P. 2004. Heat stress response in plants: a complex game with chaperones and more than twenty heat stress transcription factors. $J$ Biosci 29:471-487. 10.1007/BF02712120

Bokszczanin KL, Solanaceae Pollen Thermotolerance Initial Training Network C, and Fragkostefanakis S. 2013. Perspectives on deciphering mechanisms underlying plant heat stress response and thermotolerance. Front Plant Sci 4:315. 10.3389/fpls.2013.00315

Chidambaranathan P, Jagannadham PTK, Satheesh V, Kohli D, Basavarajappa SH, Chellapilla B, Kumar J, Jain PK, and Srinivasan R. 2018. Genome-wide analysis identifies chickpea (Cicer arietinum)

Peer] reviewing PDF | (2020:09:52717:1:1:NEW 24 Jan 2021) 
368

369

370

371

372

373

374

375

376

377

378

379

380

381

382

383

384

385

386

387

388

389

390

391

392

393

394

395

396

397

398

399

400

401

402

403

404

405

406

407

408

409

410

411

412

413

414

415

416

417

418

heat stress transcription factors (Hsfs) responsive to heat stress at the pod development stage. $J$ Plant Res 131:525-542. 10.1007/s10265-017-0948-y

Chung E, Kim KM, and Lee JH. 2013. Genome-wide analysis and molecular characterization of heat shock transcription factor family in Glycine max. J Genet Genomics 40:127-135. 10.1016/j.jgg.2012.12.002

Fragkostefanakis S, Roth S, Schleiff E, and Scharf KD. 2015. Prospects of engineering thermotolerance in crops through modulation of heat stress transcription factor and heat shock protein networks. Plant Cell Environ 38:1881-1895. 10.1111/pce.12396

Galeotti N. 2017. Hypericum perforatum (St John's wort) beyond depression: A therapeutic perspective for pain conditions. J Ethnopharmacol 200:136-146. 10.1016/j.jep.2017.02.016

Giorno F, Guerriero G, Baric S, and Mariani C. 2012. Heat shock transcriptional factors in Malus domestica: identification, classification and expression analysis. BMC Genomics 13:639. 10.1186/1471-2164-13-639

Guo J, Wu J, Ji Q, Wang C, Luo L, Yuan Y, Wang Y, and Wang J. 2008. Genome-wide analysis of heat shock transcription factor families in rice and Arabidopsis. J Genet Genomics 35:105-118. 10.1016/S1673-8527(08)60016-8

Guo M, Liu JH, Ma X, Luo DX, Gong ZH, and Lu MH. 2016. The Plant Heat Stress Transcription Factors (HSFs): Structure, Regulation, and Function in Response to Abiotic Stresses. Front Plant Sci 7:114. 10.3389/fpls.2016.00114

Guo M, Lu JP, Zhai YF, Chai WG, Gong ZH, and Lu MH. 2015. Genome-wide analysis, expression profile of heat shock factor gene family (CaHsfs) and characterisation of $\mathrm{CaHsfA} 2$ in pepper (Capsicum annuum L.). BMC Plant Biol 15:151. 10.1186/s12870-015-0512-7

Gusta LV, O'Connor BJ, and Bhatty RS. 1997. Flax (Linum usitatissimum L.) responses to chilling and heat stress on flowering and seed yield. 77:97-99. 10.4141/p95-205

Huang Y, Li MY, Wang F, Xu ZS, Huang W, Wang GL, Ma J, and Xiong AS. 2015. Heat shock factors in carrot: genome-wide identification, classification, and expression profiles response to abiotic stress. Mol Biol Rep 42:893-905. 10.1007/s11033-014-3826-X

Kotak S, Port M, Ganguli A, Bicker F, and von Koskull-Doring P. 2004. Characterization of C-terminal domains of Arabidopsis heat stress transcription factors (Hsfs) and identification of a new signature combination of plant class A Hsfs with AHA and NES motifs essential for activator function and intracellular localization. Plant J 39:98-112. 10.1111/j.1365-313X.2004.02111.x

Lausen ED, Emilsson T, and Jensen MB. 2020. Water use and drought responses of eight native herbaceous perennials for living wall systems. Urban Forestry \& Urban Greening 54:126772.

Lin Q, Jiang Q, Lin J, Wang D, Li S, Liu C, Sun C, and Chen K. 2015. Heat shock transcription factors expression during fruit development and under hot air stress in Ponkan (Citrus reticulata Blanco cv. Ponkan) fruit. Gene 559:129-136. 10.1016/j.gene.2015.01.024

Lin YX, Jiang HY, Chu ZX, Tang XL, Zhu SW, and Cheng BJ. 2011. Genome-wide identification, classification and analysis of heat shock transcription factor family in maize. BMC Genomics 12:76. 10.1186/1471-2164-12-76

Livak KJ, and Schmittgen TD. 2001. Analysis of relative gene expression data using real-time quantitative PCR and the 2(-Delta Delta C(T)) Method. Methods 25:402-408. $10.1006 /$ meth.2001.1262

Miller G, and Mittler R. 2006. Could heat shock transcription factors function as hydrogen peroxide sensors in plants? Ann Bot 98:279-288. 10.1093/aob/mcl107

Mittler R, Finka A, and Goloubinoff P. 2012. How do plants feel the heat? Trends Biochem Sci 37:118125. 10.1016/j.tibs.2011.11.007

Nahrstedt A, and Butterweck V. 2010. Lessons learned from herbal medicinal products: the example of St. John's Wort (perpendicular). J Nat Prod 73:1015-1021. 10.1021/np1000329

Nishizawa-Yokoi A, Yoshida E, Yabuta Y, and Shigeoka S. 2009. Analysis of the regulation of target genes by an Arabidopsis heat shock transcription factor, HsfA2. Biosci Biotechnol Biochem 73:890-895. 10.1271/bbb.80809

Peer] reviewing PDF | (2020:09:52717:1:1:NEW 24 Jan 2021) 
419

420

421

422

423

424

425

426

427

428

429

430

431

432

433

434

435

436

437

438

439

440

441

442

443

444

445

446

447

448

449

450

451

452

453

454

455

456

457

458

459

460

461

462

463

464

465

466

467

Nover L, Bharti K, Doring P, Mishra SK, Ganguli A, and Scharf KD. 2001. Arabidopsis and the heat stress transcription factor world: how many heat stress transcription factors do we need? Cell Stress Chaperones 6:177-189. 10.1379/1466-1268(2001)006<0177:aathst $>2.0 . c 0 ; 2$

Prandl R, Hinderhofer K, Eggers-Schumacher G, and Schoffl F. 1998. HSF3, a new heat shock factor from Arabidopsis thaliana, derepresses the heat shock response and confers thermotolerance when overexpressed in transgenic plants. Mol Gen Genet 258:269-278. 10.1007/s004380050731

Sadok W, Lopez JR, and Smith KP. 2020. Transpiration increases under high temperature stress: potential mechanisms, trade-offs and prospects for crop resilience in a warming world. Plant Cell Environ. $10.1111 /$ pce. 13970

Saha D, Mukherjee P, Dutta S, Meena K, Sarkar SK, Mandal AB, Dasgupta T, and Mitra J. 2019. Genomic insights into HSFs as candidate genes for high-temperature stress adaptation and gene editing with minimal off-target effects in flax. Sci Rep 9:5581. 10.1038/s41598-019-41936-1

Samakovli D, Ticha T, Vavrdova T, Ovecka M, Luptovciak I, Zapletalova V, Kucharova A, Krenek P, Krasylenko Y, Margaritopoulou T, Roka L, Milioni D, Komis G, Hatzopoulos P, and Samaj J. 2020. YODA-HSP90 Module Regulates Phosphorylation-Dependent Inactivation of SPEECHLESS to Control Stomatal Development under Acute Heat Stress in Arabidopsis. Mol Plant 13:612-633. 10.1016/j.molp.2020.01.001

Scharf KD, Berberich T, Ebersberger I, and Nover L. 2012. The plant heat stress transcription factor (Hsf) family: structure, function and evolution. Biochim Biophys Acta 1819:104-119. 10.1016/j.bbagrm.2011.10.002

Schultheiss J, Kunert O, Gase U, Scharf KD, Nover L, and Ruterjans H. 1996. Solution structure of the DNA-binding domain of the tomato heat-stress transcription factor HSF24. Eur J Biochem 236:911-921. 10.1111/j.1432-1033.1996.00911.x

Skyba M, Petijova L, Kosuth J, Koleva DP, Ganeva TG, Kapchina-Toteva VM, and Cellarova E. 2012. Oxidative stress and antioxidant response in Hypericum perforatum L. plants subjected to low temperature treatment. J Plant Physiol 169:955-964. 10.1016/j.jplph.2012.02.017

Song L, Guanter L, Guan K, You L, Huete A, Ju W, and Zhang Y. 2018. Satellite sun-induced chlorophyll fluorescence detects early response of winter wheat to heat stress in the Indian IndoGangetic Plains. Glob Chang Biol 24:4023-4037. 10.1111/gcb.14302

Thirumalaikumar VP, Gorka M, Schulz K, Masclaux-Daubresse C, Sampathkumar A, Skirycz A, Vierstra RD, and Balazadeh S. 2020. Selective autophagy regulates heat stress memory in Arabidopsis by NBR1-mediated targeting of HSP90 and ROF1. Autophagy:1-16. 10.1080/15548627.2020.1820778

Wang P, Song H, Li C, Li P, Li A, Guan H, Hou L, and Wang X. 2017. Genome-Wide Dissection of the Heat Shock Transcription Factor Family Genes in Arachis. Front Plant Sci 8:106. 10.3389/fpls.2017.00106

Wang W, Vinocur B, Shoseyov O, and Altman A. 2004. Role of plant heat-shock proteins and molecular chaperones in the abiotic stress response. Trends Plant Sci 9:244-252. 10.1016/j.tplants.2004.03.006

Yang X, Zhu W, Zhang H, Liu N, and Tian S. 2016. Heat shock factors in tomatoes: genome-wide identification, phylogenetic analysis and expression profiling under development and heat stress. PeerJ 4:e1961. 10.7717/peerj.1961

Zhang J, Liu B, Li J, Zhang L, Wang Y, Zheng H, Lu M, and Chen J. 2015. Hsf and Hsp gene families in Populus: genome-wide identification, organization and correlated expression during development and in stress responses. BMC Genomics 16:181. 10.1186/s12864-015-1398-3

Zhou W, Wang Y, Li B, Petijová L, Hu S, Zhang Q, Niu J, Wang D, Wang S, Dong Y, Čellárová E, and Wang Z. 2020. Whole genome sequence data of Hypericum perforatum and functional characterization of melatonin biosynthesis by $\mathrm{N}$ - acetylserotonin $\mathrm{O}$ - methyltransferase. Journal of Pineal Research. e12709. 10.1111/jpi.12709

Peer] reviewing PDF | (2020:09:52717:1:1:NEW 24 Jan 2021) 
468

469

470

471

472

473

474

475

476

477

478

479

Zhou W, Wang S, Yang L, Sun Y, Zhang Q, Li B, Wang B, Li L, Wang D, and Wang Z. 2019. Reference genes for qRT-PCR normalisation in different tissues, developmental stages, and stress conditions of Hypericum perforatum. PeerJ 7:e7133. 10.7717/peerj.7133

Zhou W, Zhang Q, Sun Y, Yang L and Wang Z. 2020. Genome-wide identification and characterization of R2R3-MYB family in Hypericum perforatum under diverse abiotic stresses. Int J Biol Macromol 145:341-354. 10.1016/j.ijbiomac.2019.12.100

Zhu P, Zhuang Q, Archontoulis SV, Bernacchi C, and Muller C. 2019. Dissecting the nonlinear response of maize yield to high temperature stress with model-data integration. Glob Chang Biol 25:24702484. $10.1111 / \mathrm{gcb} .14632$

Zobayed SM, Afreen F, and Kozai T. 2005. Temperature stress can alter the photosynthetic efficiency and secondary metabolite concentrations in St. John's wort. Plant Physiol Biochem 43:977-984.

480 


\section{Table $\mathbf{1}$ (on next page)}

The HSF genes identifed from the H. perforatum.

Notes: MW (kDa): Molecular weight in kilo Dalton; pl, isoelectric point; n.c.r., total number of negatively charged residues (Asp +Glu); p.c.r., total number of positively charged residues (Arg +Lys); I.I., instability index; A.I., aliphatic index; GRAVY, grand average of hydropathicity. 


\begin{tabular}{|c|c|c|c|c|c|c|c|c|c|c|c|c|}
\hline \multirow{2}{*}{$\begin{array}{l}\text { Gene } \\
\text { Name }\end{array}$} & \multirow{2}{*}{$\begin{array}{l}\text { Transcript } \\
\text { ID }\end{array}$} & \multicolumn{3}{|c|}{ Length } & \multirow{2}{*}{$\begin{array}{c}\text { MW } \\
\text { (kDa) }\end{array}$} & \multirow[b]{2}{*}{ pI } & \multirow[b]{2}{*}{ n.c.r. (\%) } & \multirow[b]{2}{*}{ p.c.r. (\%) } & \multirow[b]{2}{*}{ I.I. } & \multirow[b]{2}{*}{ Stability } & \multirow[b]{2}{*}{ A.I. } & \multirow[b]{2}{*}{ GRAVY } \\
\hline & & Protein & CDS & Gene & & & & & & & & \\
\hline HpHSF01 & $\begin{array}{l}\text { HperS113g } \\
0097\end{array}$ & 293 & 882 & 1058 & 32.23 & 5.05 & $43(14.7 \%)$ & $35(11.9 \%)$ & 57.40 & unstable & 75.26 & -0.523 \\
\hline HpHSF02 & $\begin{array}{l}\text { HperS020g } \\
0043\end{array}$ & 381 & 1146 & 1687 & 43.75 & 5.51 & $59(15.5 \%)$ & $48(12.6 \%)$ & 59.80 & unstable & 71.55 & -0.752 \\
\hline $\mathrm{HpHSF03}$ & $\begin{array}{l}\text { HperS219g } \\
0006\end{array}$ & 327 & 984 & 1842 & 37.9 & 7.29 & $36(11.0 \%)$ & $36(11.0 \%)$ & 47.69 & unstable & 72.14 & -0.660 \\
\hline $\mathrm{HpHSF04}$ & $\begin{array}{l}\text { HperS024g } \\
0021\end{array}$ & 222 & 669 & 1798 & 25.95 & 7.72 & $34(15.3 \%)$ & $35(15.8 \%)$ & 52.96 & unstable & 73.24 & -0.796 \\
\hline HpHSF05 & $\begin{array}{l}\text { HperS024g } \\
0048\end{array}$ & 196 & 591 & 2959 & 22.47 & 6.85 & $31(15.8 \%)$ & $31(15.8 \%)$ & 46.57 & unstable & 69.08 & -0.747 \\
\hline HpHSF06 & $\begin{array}{l}\text { HperS245g } \\
0169\end{array}$ & 226 & 681 & 3135 & 25.88 & 6.86 & $34(15.0 \%)$ & $34(15.0 \%)$ & 48.26 & unstable & 69.38 & -0.737 \\
\hline HpHSF07 & $\begin{array}{l}\text { HperS025g } \\
0041\end{array}$ & 434 & 1305 & 1764 & 48.47 & 5.22 & $64(14.7 \%)$ & $47(10.8 \%)$ & 58.65 & unstable & 76.18 & -0.577 \\
\hline HpHSF08 & $\begin{array}{l}\text { HperS254g } \\
0338\end{array}$ & 376 & 1131 & 1300 & 42.04 & 5.67 & $45(12.0 \%)$ & $39(10.4 \%)$ & 66.18 & unstable & 64.84 & -0.655 \\
\hline HpHSF09 & $\begin{array}{l}\text { HperS338g } \\
0001\end{array}$ & 330 & 993 & 1169 & 36.57 & 5.67 & $43(13.0 \%)$ & $38(11.5 \%)$ & 50.96 & unstable & 60.24 & -0.600 \\
\hline HpHSF10 & $\begin{array}{l}\text { HperS346g } \\
0011\end{array}$ & 428 & 1287 & 1897 & 48.01 & 4.91 & $66(15.4 \%)$ & $44(10.3 \%)$ & 56.52 & unstable & 67.64 & -0.642 \\
\hline$H p H S F 11$ & $\begin{array}{l}\text { HperS346g } \\
0247\end{array}$ & 324 & 975 & 1054 & 37.51 & 5.91 & $45(13.9 \%)$ & $38(11.7 \%)$ & 57.46 & unstable & 59.85 & -0.813 \\
\hline HpHSF 12 & $\begin{array}{l}\text { HperS362g0 } \\
014\end{array}$ & 409 & 1230 & 1464 & 46.16 & 5.02 & $65(15.9 \%)$ & $43(10.5 \%)$ & 57.83 & unstable & 65.99 & -0.745 \\
\hline HpHSF 13 & $\begin{array}{l}\text { HperS388g0 } \\
082\end{array}$ & 403 & 1212 & 2262 & 46.49 & 4.79 & $71(17.6 \%)$ & $46(11.4 \%)$ & 47.52 & unstable & 65.56 & -0.764 \\
\hline HpHSF14 & $\begin{array}{l}\text { HperS398g0 } \\
019\end{array}$ & 195 & 588 & 1981 & 22.35 & 8.86 & $26(13.3 \%)$ & $30(15.4 \%)$ & 63.69 & unstable & 58.10 & -0.818 \\
\hline HpHSF15 & $\begin{array}{l}\text { HperS042g0 } \\
257\end{array}$ & 248 & 747 & 1956 & 27.78 & 5.78 & $40(16.1 \%)$ & $37(14.9 \%)$ & 46.02 & unstable & 61.33 & -0.817 \\
\hline HpHSF16 & $\begin{array}{l}\text { HperS434g0 } \\
151\end{array}$ & 501 & 1506 & 2644 & 54.91 & 4.87 & $64(12.8 \%)$ & $42(8.4 \%)$ & 59.15 & unstable & 67.60 & -0.608 \\
\hline HpHSF17 & $\begin{array}{l}\text { HperS044g0 } \\
424\end{array}$ & 483 & 1452 & 2149 & 53.92 & 4.99 & $63(13.0 \%)$ & $42(8.7 \%)$ & 51.71 & unstable & 74.66 & -0.533 \\
\hline HpHSF18 & $\begin{array}{l}\text { HperS443g0 } \\
073\end{array}$ & 397 & 1194 & 1521 & 44.95 & 4.94 & $63(15.9 \%)$ & $41(10.3 \%)$ & 62.46 & unstable & 70.48 & -0.723 \\
\hline HpHSF19 & $\begin{array}{l}\text { HperS006g0 } \\
172\end{array}$ & 188 & 567 & 1981 & 21.72 & 8.54 & $25(13.3 \%)$ & $28(14.9 \%)$ & 52.32 & unstable & 54.52 & -0.797 \\
\hline HpHSF20 & $\begin{array}{l}\text { HperS064g0 } \\
032\end{array}$ & 455 & 1368 & 1950 & 51.78 & 5.91 & $64(14.1 \%)$ & $57(12.5 \%)$ & 62.11 & unstable & 71.12 & -0.644 \\
\hline$H p H S F 21$ & $\begin{array}{l}\text { HperS068g0 } \\
017\end{array}$ & 495 & 1488 & 2278 & 54.66 & 4.96 & $65(13.1 \%)$ & $45(9.1 \%)$ & 56.75 & unstable & 67.39 & -0.650 \\
\hline$H p H S F 22$ & $\begin{array}{l}\text { HperS079g0 } \\
626\end{array}$ & 270 & 813 & 1208 & 31.63 & 6.33 & $33(12.2 \%)$ & $28(10.4 \%)$ & 47.61 & unstable & 64.59 & -0.826 \\
\hline$H p H S F 23$ & $\begin{array}{l}\text { HperS091g0 } \\
277\end{array}$ & 363 & 1092 & 1604 & 41.62 & 5.39 & $57(15.7 \%)$ & $43(11.8 \%)$ & 61.47 & unstable & 69.53 & -0.784 \\
\hline
\end{tabular}




\section{Table 2 (on next page)}

Conserved domains and motifs of HpHSF proteins.

Notes: DBD: DNA-bind domain; OD: heptad repeat A (N-terminus) or B (C-terminus) domain; NLS: nuclear localization signal; NES: nuclear export signal; AHA: aromatic and hydrophobic amino acid residues embedded in an acidic context; RD: repressor domain. 
1

\begin{tabular}{|l|l|c|c|c|c|c|c|c|}
\hline Gene Name & Group & DBD & OD & NLS & NES & AHA & RD & RD+NLS \\
\hline HpHSF01 & B2 & $12-130$ & $159-209$ & - & - & - & - & $238-265$ \\
HpHSF02 & A6 & $34-136$ & $141-223$ & $224-235$ & $360-378$ & $329-342$ & - & - \\
HpHSF03 & B4 & $9-109$ & $174-211$ & - & - & - & - & $244-267$ \\
HpHSF04 & B3 & $14-114$ & $129-185$ & - & - & - & $194-208$ & - \\
HpHSF05 & B3 & $19-89$ & $112-159$ & - & - & - & - & - \\
HpHSF06 & B3 & $17-117$ & $125-181$ & $209-224$ & - & - & $190-204$ & - \\
HpHSF07 & A5 & $11-107$ & $125-197$ & $197-215$ & - & - & - & - \\
HpHSF08 & С & $38-136$ & $156-220$ & - & - & - & - & - \\
HpHSF09 & С & $9-107$ & $128-192$ & $197-212$ & - & - & - & - \\
HpHSF10 & A3 & $17-120$ & $137-205$ & $209-227$ & - & - & - & - \\
HpHSF11 & A4 & $10-123$ & $136-216$ & - & - & - & - & - \\
HpHSF12 & A4 & $6-119$ & $122-202$ & $205-225$ & $392-407$ & $374-390$ & - & - \\
HpHSF13 & A8 & $15-116$ & $142-213$ & $213-222$ & $381-395$ & - & - & - \\
HpHSF14 & B5 & $10-128$ & $149-190$ & - & - & - & - & - \\
HpHSF15 & B1 & $1-101$ & $144-193$ & - & - & - & $226-237$ & - \\
HpHSF16 & A1 & $23-119$ & $129-222$ & $223-241$ & $480-495$ & $433-451$ & - & - \\
HpHSF17 & A1 & $5-101$ & $102-195$ & $198-216$ & $462-477$ & $412-430$ & - & - \\
HpHSF18 & A4 & $6-119$ & $122-202$ & $205-225$ & $380-395$ & $322-378$ & - & - \\
HpHSF19 & B5 & $9-120$ & $142-183$ & - & - & - & - & - \\
HpHSF20 & A3 & $29-158$ & $174-242$ & $246-264$ & - & - & - & - \\
HpHSF21 & A1 & $16-112$ & $122-215$ & $215-233$ & $473-488$ & $426-444$ & - & - \\
HpHSF22 & B4 & $9-109$ & $177-224$ & - & - & - & - & $247-270$ \\
HpHSF23 & A6 & $30-132$ & $137-219$ & $220-242$ & $342-360$ & $312-325$ & - & - \\
\hline
\end{tabular}


Figure 1

\section{Multiple sequence alignment of the DBD domains of 23 members of the HSF protein}

family.

\section{Three $\alpha$ - helices and four $\beta$ - sheets were presented in the region.}

Consensus
HpHSF01
HpHSF02
HpHSF03
HpHSF04
HpHSF05
HpHSF06
HpHSF07
HpHSF08
HpHSF09
HpHSF10
HpHSF11
HpHSF12
HpHSF13
HpHSF14
HpHSF15
HpHSF16
HpHSF17
HpHSF18
HpHSF19
HpHSF20
HpHSF21
HpHSF22
HpHSF23

Sequence Logo

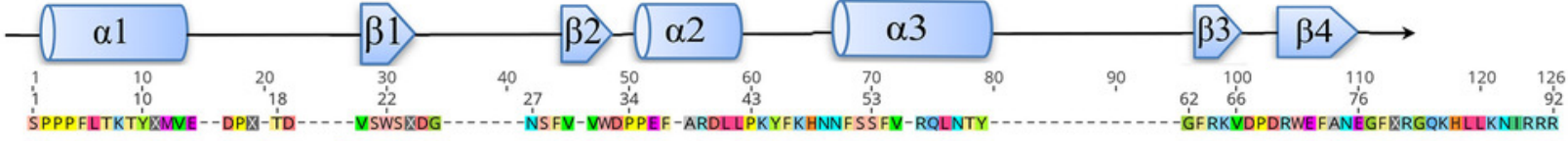

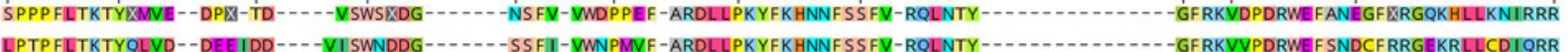

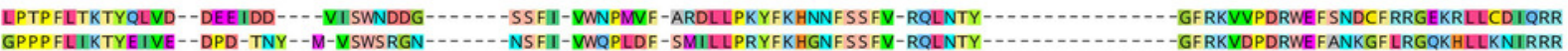
VPAP FILTKTYOLVD--DPL-TDH--II-VSWGDDE-- - - ATFII-WWRPPEF-ARDLIPNYFKHCNFSSFV-ROLNTY-- -

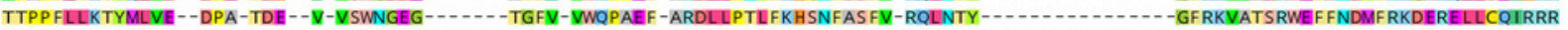

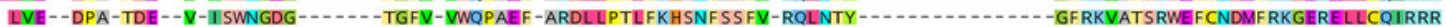

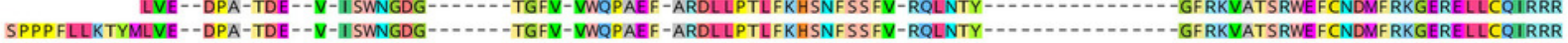
SPPP FLLLKTYMLVE--DPA-TDE--V-I SWNGDG--1

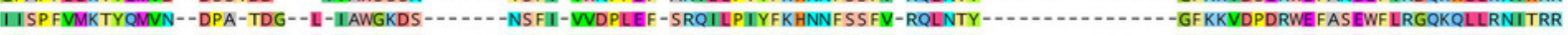
TVAP FVMKTYOMVN--DPA-TDR--I-ISWGKAD----NSFF-NVDPLDF-SHRIIPIYYFKHNNFSSFV-RQLNTY---

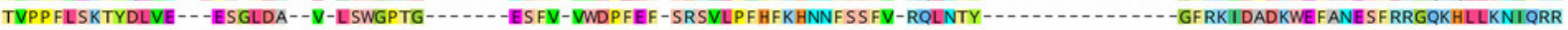
SPAP FLVKTYEMVD--DYS-TDH--II-VSWSSSG-----CSFV-WWDPPEF-SQDLLPKYFKHNNFSSFV-RQLNTY--

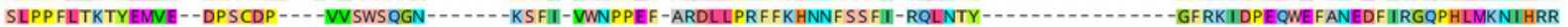
SVAP FEKKCYEMV---DDS STDA-- $v$-ISWSGDDS-- - - SSFV-IWDSTEF-SVRLLPEYFKHSNFSS FII-ROLNIY-- - - SPAP FEAKTYDILEEGDRGGGDGNRRIVSWNEDG-- - - DGFVV-WSPADF-SELMLPRYFKHNNFSSFV-ROLNTY--

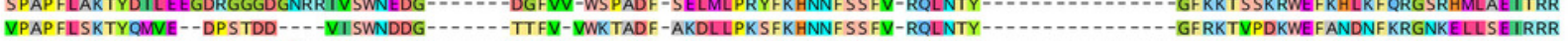

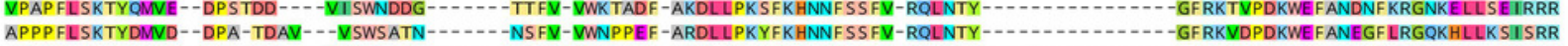

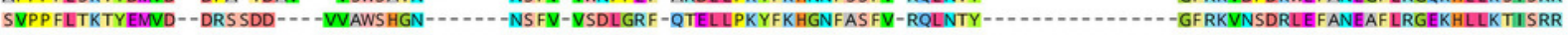

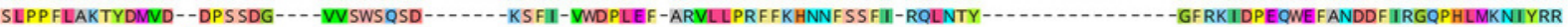

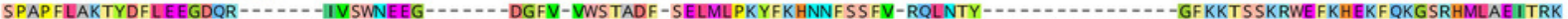
MVPP FLSKTYDLVE--DPALDG - - -VLSWGRRGELRGLGPTRVLQVRP PPLLQAQQLLOLRPPAQHLPQLLMRYLPTYLYNRQRRDECFEPLVQGFRKIDTDKWEFANESFKRGKKOLLKNIQRR

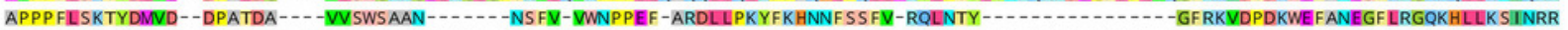
VPAP FITKTYQLVD--DPL-TDH--II-VSWGDDE--- -ATFII-WWRPPEF-ARDLLPNYFKHCNFSSFV-RQLNTY--

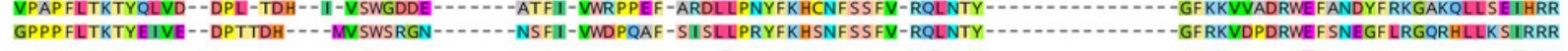

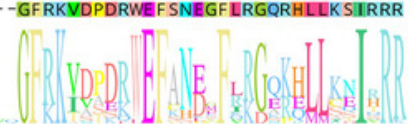




\section{Figure 2}

Multiple sequence alignment of the HR-A/B regions of 23 members of the HSF protein family.

The annotations at the top describe the location and boundaries of the HR-A core, insert, and $H R-B$ region within the HR-A/B region.

Class A

Class C

Class B

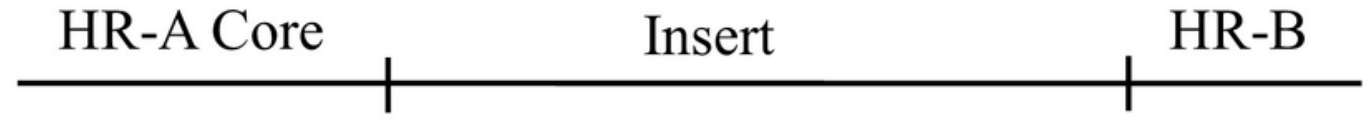

HPHSF 10 HpHSF 20 HpHSFO2 HpHSF 23 HPHSF 13 HPHSF 17 HPHSF 16 HpHSF 21 HpHSF 12 HpHSF 18 HPHSF 11 HpHSF 07 HPHSF0 8 HPHSFO 9 HpHSFO 3 HpHSF 22 HPHSFO 5 HPHSFO 6 HpHSFO 4 HPHSF 15 HPHSFO 1 HpHSF 14 HPHSF 19

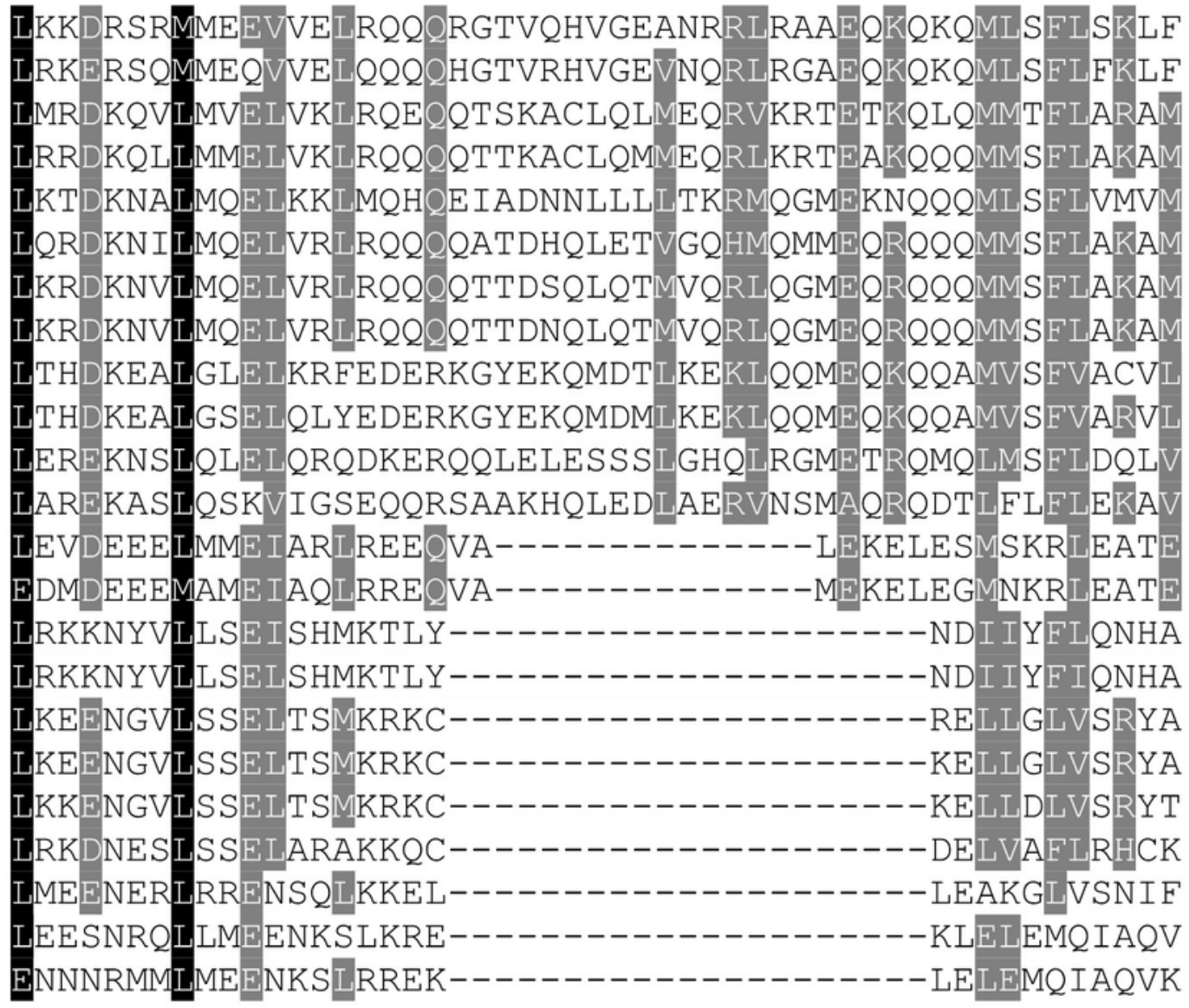




\section{Figure 3}

Phylogenetic tree of HSF proteins from H. perforatum (Hp), Capsicum annuum L. (Ca), Vitis vinifera $L$. $(\mathrm{V})$, and $A$. thaliana (At).

The full-length of amino acid sequences of HSF proteins in the four species were used to construct the phylogenetic tree using MEGA 6, the statistical method used was the NeighborJoining (NJ) method, and the test of phylogeny was based on the bootstrap method with 1000 bootstrap replicates and pairwise deletion. The amino acid substitution model used was the p-distance model. Subclass numbers of Arabidopsis, pepper, and grape are listed. 


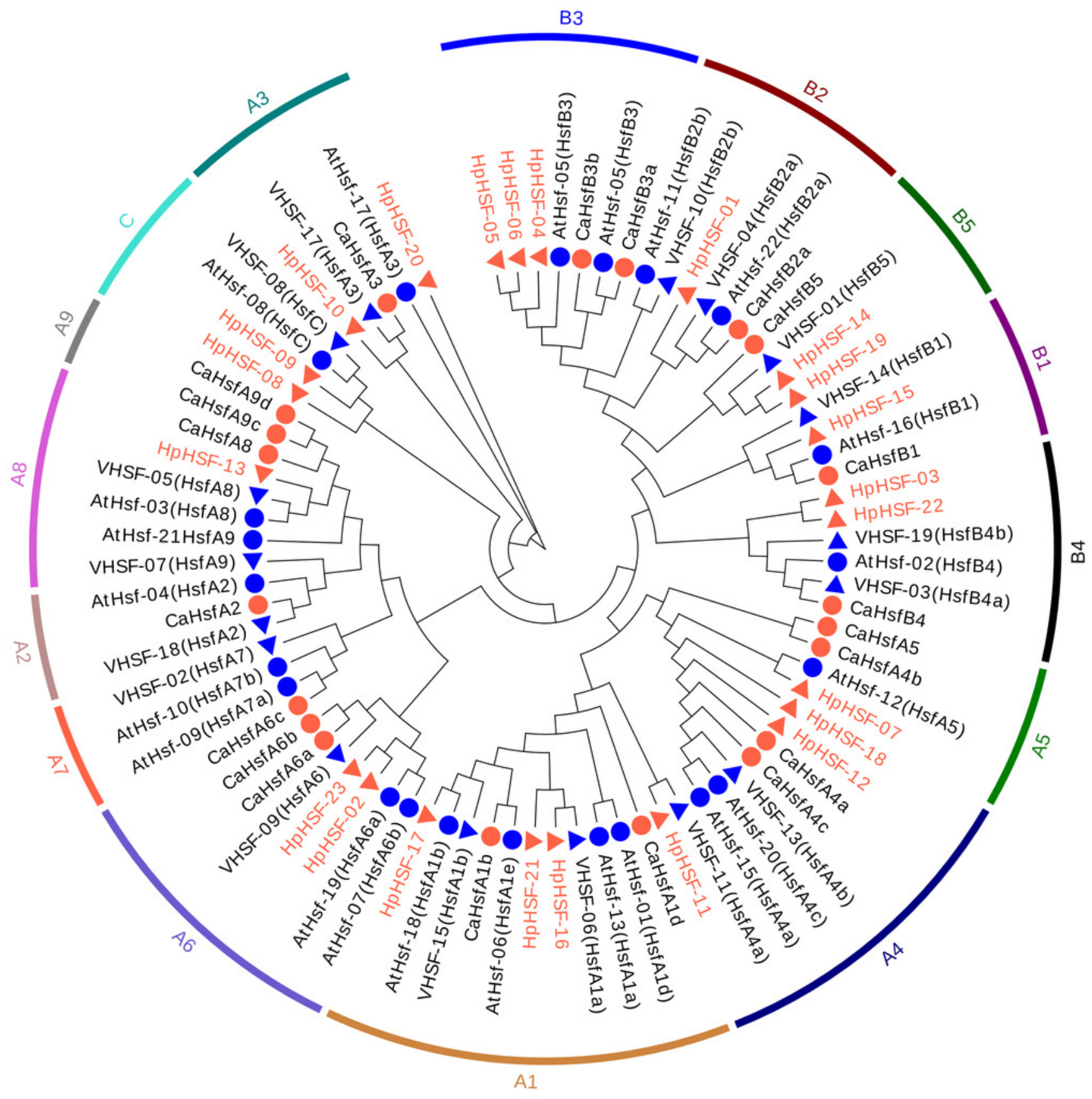


Figure 4

Gene structure (A) and conserved motifs (B) of HpHSF family members.

(A) blank box, Grey box and black line were represented CDS, upstream/ downstream and intron, respectively. The number 0,1 , and 2 on the black line were intron phase. (B) DBD, OD (HR-A/B), RD, NLS, AHA and NES motifs of HpHSF members were identified by Heatster. The motifs were annotated and exhibited in different colored boxes.

A

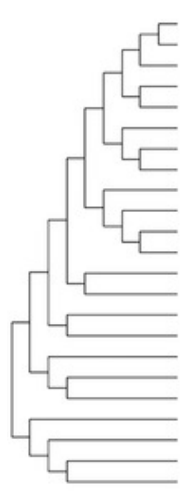

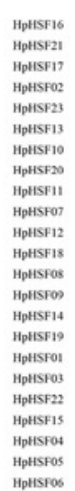

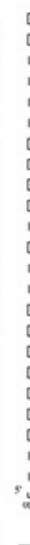

Legend:

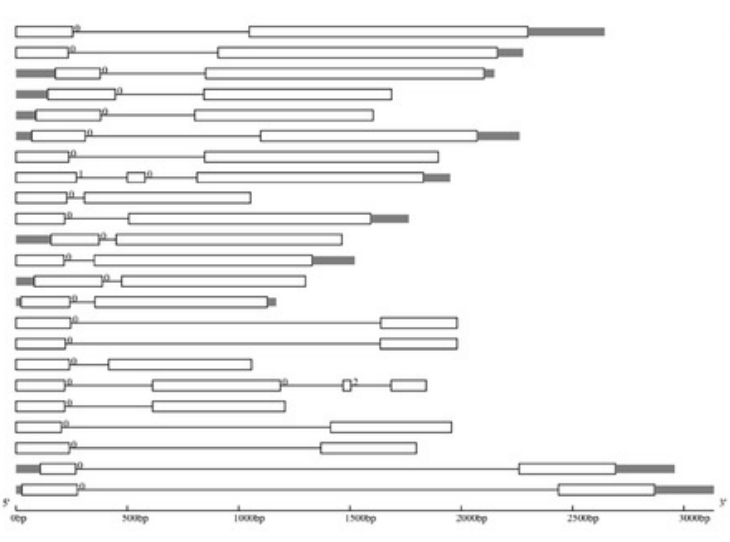

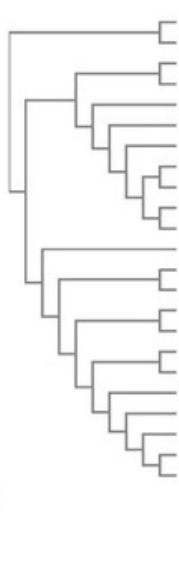

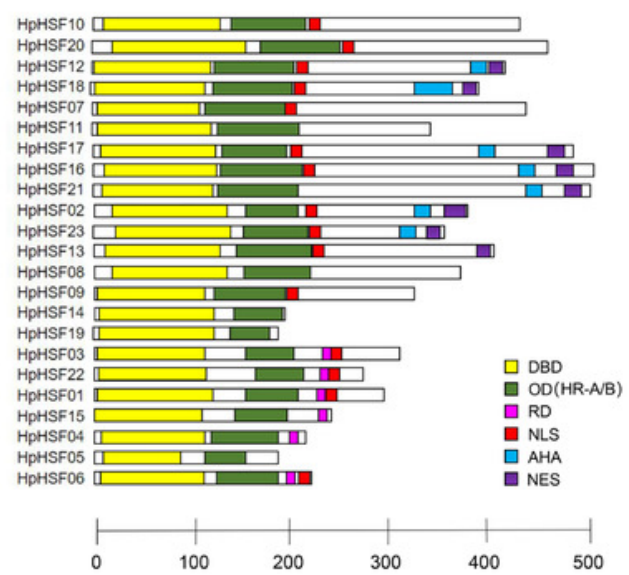


Figure 5

Modelling of HpHSF family members, A Drosophila HSF was used as a template.

Templates corresponding to SMTL ID: 1hkt.1. The HpHSFs shared approximately $40 \%$

sequence similarity and 30\% query coverage. The start position of the $\alpha 1$ DBD domain was notated by 1 . 


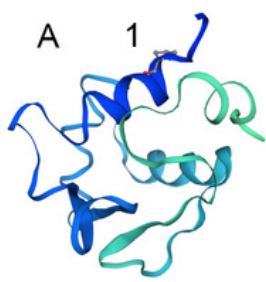

HpHSF1

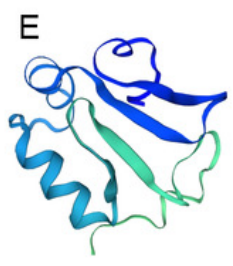

HpHSF5

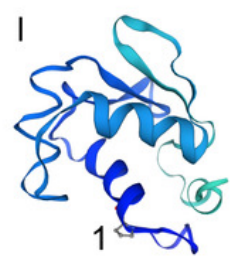

HpHSF9

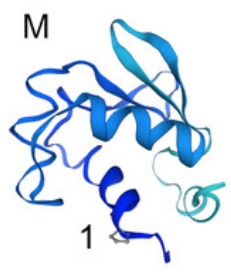

HpHSF13

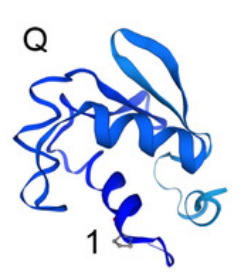

HpHSF17

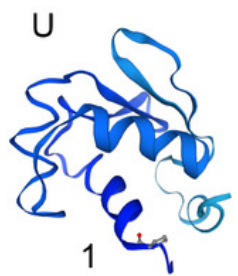

HpHSF21

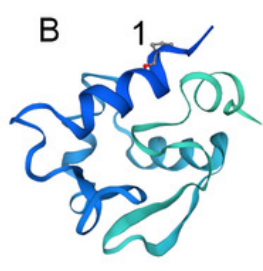

HpHSF2

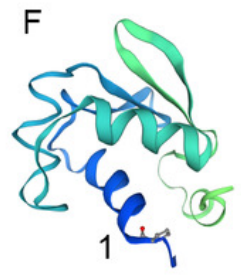

HpHSF6

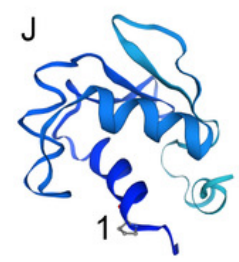

HpHSF10

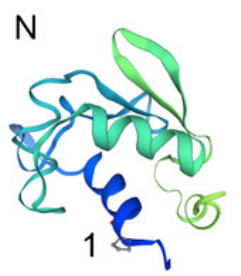

HpHSF14

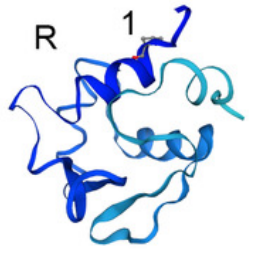

HpHSF18

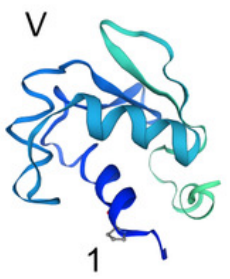

HpHSF22

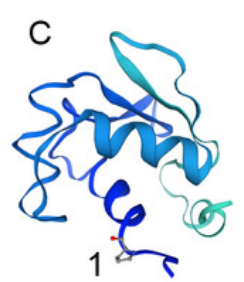

HpHSF3

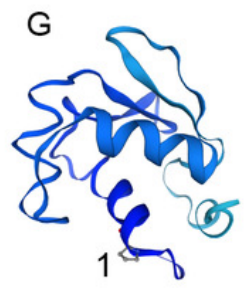

HpHSF7

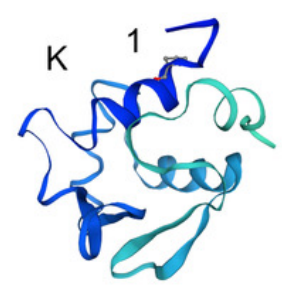

HpHSF11

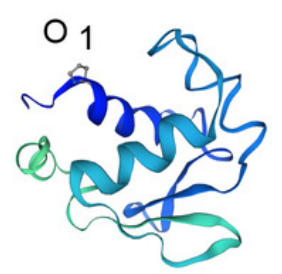

HpHSF15

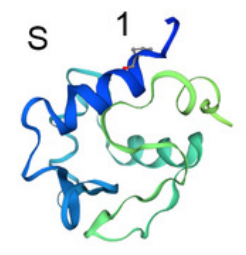

HpHSF19

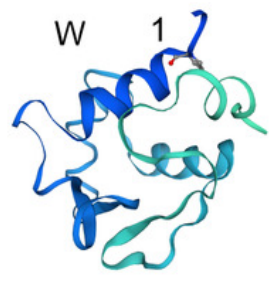

HpHSF23

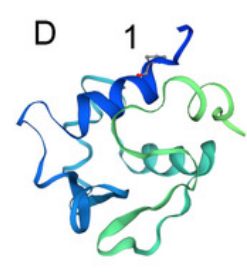

HpHSF4

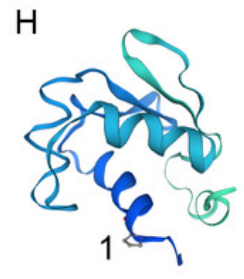

HpHSF8

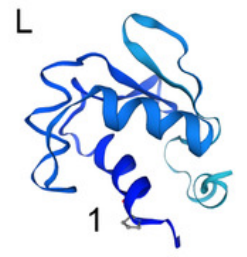

HpHSF12

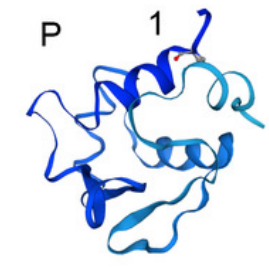

HpHSF16

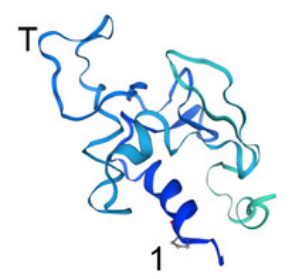

HpHSF20 
Figure 6

Number of HpHSF genes containing various cis-acting elements.

The graph was generated based on the presence of cis-acting elements responsive tospecific processes/elicitors/conditions ( $x$-axis) in HSF gene family members (y-axis).

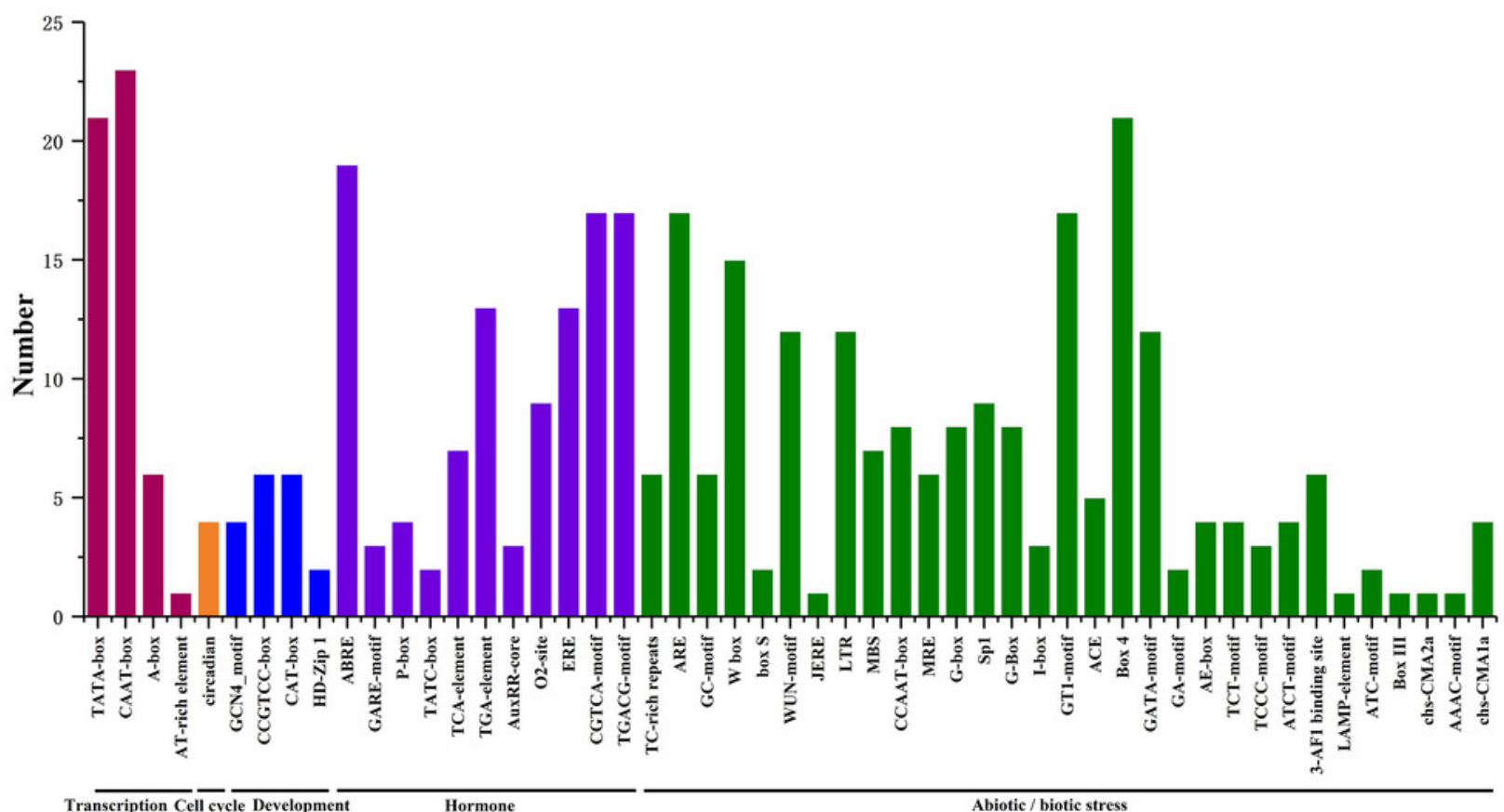


Figure 7

Heat map representation and hierarchical clustering of HpHSF genes in flower, leaf, root, stem.

The expression values were calculated by fragments per kilobase of exon model per million mapped (FPKM). 


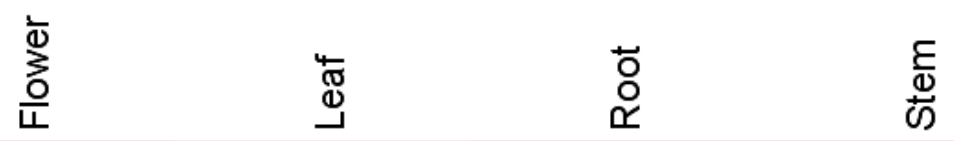

HpHSF16

HpHSF21

HpHSF17

HpHSF11

HpHSF12

HpHSF18

HpHSF07

HpHSF02

HpHSF23

HpHSF13

HpHSF10

HpHSF20

HpHSF04

HpHSF05

HpHSF06

HpHSF01

HpHSF14

HpHSF19

HpHSF15

HpHSF03

HpHSF22

HpHSF09 
Figure 8

Relative gene expression of HpHSFs analyzed by qRT-PCR response to heat stress treatment.

qRT-PCR data was normalized using Hypericum perforatum Actin 2 gene and are shown relative to $0 \mathrm{~h}$. X-axes are time course $(0 \mathrm{~h}, 1 \mathrm{~h}, 3 \mathrm{~h}, 6 \mathrm{~h}$ and $12 \mathrm{~h})$ and y-axes are scales of relative expression level. All Data represent means \pm SD of three independent replicates. Statistical significance was analyzed by one way ANOVA (*, P <0.05; **, P $<0.01$; ***, P < $0.001)$. 


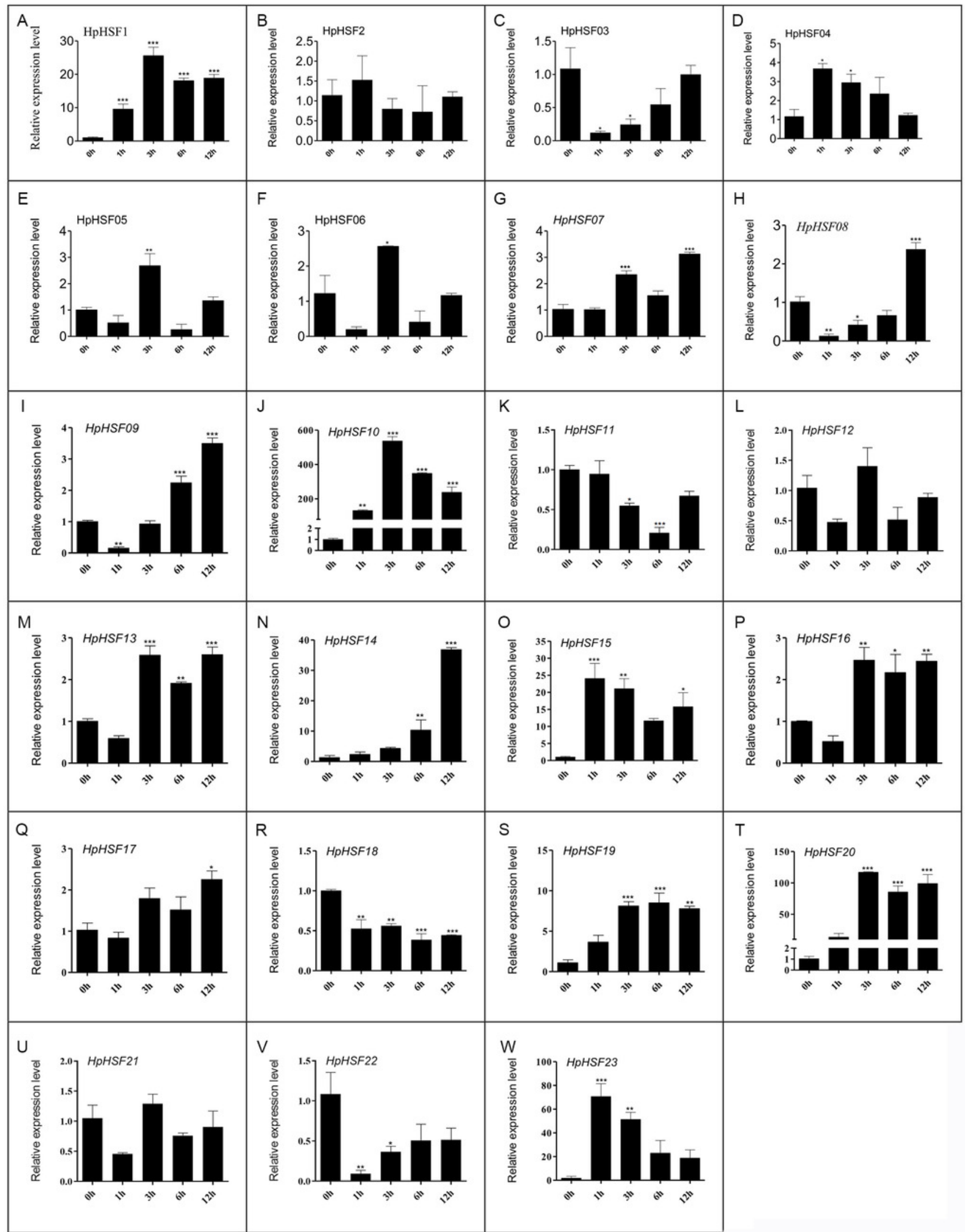

